\title{
Local Solutions \\ to Quasilinear Parabolic Equations without Growth Restrictions
}

\author{
V. Pluschke
}

\begin{abstract}
The paper deals with quasilinear parabolic boundary value problems where the nonlinear coefficients and right-hand side are defined with respect to the unknown function $u=u(x ; t)$ only in a neighbourhood of the initial function. The quasilinear parabolic problem is approximated by linear elliptic problems by means of semidiscretization in time. It is proved that the approximations converge uniformly although the data are not continuous functions, and the limit turns out to be the weak solution of the parabolic problem for sufficiently small time $t$. The crucial points of the paper are $L_{\infty}$-estimates to ensure that the approximations belong to the domain of non-linearities and uniform estimates of the discrete time derivatives in a Lebesgue space in order to obtain uniform convergence.
\end{abstract}

Keywords: Semidiscretization in time, quasilinear parabolic equations, local solutions, $L_{\infty}$ - estimates

AMS subject classification: Primary $35 \mathrm{~K} \mathrm{20}$, secondary $35 \mathrm{~K} 55,65 \mathrm{M} 20$

\section{Introduction}

In this paper we want to prove existence of a weak solution to the parabolic initial boundary value problem

$$
\begin{aligned}
D_{t} u+A(t, u) u & =f(x, t, u) & & \text { in } Q_{T} \\
\cdot u(x, t) & =0 & & \text { on } \Gamma \\
u(x, 0) & =U_{0}(x) & & \text { inenskip } G
\end{aligned}
$$

where

$$
\begin{aligned}
A(t, v) u & =-\sum_{i, k=1}^{N} \frac{\partial}{\partial x_{k}}\left(a_{i k}(x, t, v) \frac{\partial u}{\partial x_{i}}\right)+\sum_{i=1}^{N} a_{i}(x, t, v) \frac{\partial u}{\partial x_{i}} \\
D_{t} u & =\frac{\partial u}{\partial t}
\end{aligned}
$$

by means of approximation by the Rothe method. Here we denote. by $G \subset \mathbb{R}^{N}(N \geq 2)$ a simply connected, bounded domain with boundary $\partial G \in C^{1}, I=[0, T], Q_{T}=G \times I$ and $\Gamma=\partial G \times I$.

V. Pluschke: Martin-Luther-Univ. Halle - Wittenberg, Dept. Math', D - 06099 Halle (Saale) 
We point out that the coefficients of the operator $A$ depend on the unknown function $u$. The Rothe method was applied to rather general nonlinear differential equations especially in the papers of Kacur (see [2 - 5]). In these papers convergence of the approximations usually is obtained by means of compactness arguments. In the present case we will estimate the convergence order and error of the Rothe approximations. In some papers (see, e.g., [3]) the Cauchy sequences of Rothe approximations were estimated for a monotone operator $A$. We will do this without assuming monotonicity of $A$. Our proof of convergence is based on an a priori estimate of the time derivative derived by means of some nonlinear Gronwall lemma.

Furthermore, we present $L_{\infty}$-estimates for the approximations to the quasilinear problem (1.1) - (1.4). This allows to omit any growth restriction of the coefficients and the right-hand side with respect to $u$. In addition, assumptions like Lipschitz condition need only be supposed on a bounded set. For the proof of the $L_{\infty}$-estimates we use the technique of Moser [7] and Alikakos [1], where estimates in $L_{\infty}$-norms are obtained by a limit process $p \rightarrow \infty$. This technique was used by the author to deal with the Rothe method for semilinear parabolic problems in [9] and problems with degenerating coefficient in [10].

\section{Preliminaries}

In the following $\|\cdot\|_{p}$ denotes the norm in $L_{p}(G)$ and $\langle\cdot, \cdot\rangle$ the duality between $L_{p}(G)$ and $L_{p^{\prime}}(G)\left(\frac{1}{p}+\frac{1}{p}^{\prime}=1\right)$. $W^{1, p}(G)$ and $W_{0}^{1, p}(G)$ are the usual Sobolev spaces, the last one being normed by $\|u\|_{1, p}=\|\nabla u\|_{p}$. For $t \in I$ and $v \in C(\bar{G})$ the operator $A(t, v)$ from (1.4) generates a bilinear form on $W_{0}^{1, p}(G) \times W_{0}^{1, p^{\prime}}(G)$ denoted by $A_{(t, v)}(\cdot, \cdot)$. Moreover, we use $C(I, V), C^{0,1}(I, V)$ and $L_{p}(I, V)$ for the sets of continuous, Lipschitz continuous, and $L_{p}$-integrable mappings $I \rightarrow V$, respectively. By $c$ we denote generic constants which may be different on different places but are independent of the subdivision and of $p$ if it is variable. Furthemore, by $\mathbb{N}$ and $N_{0}$ we denote the sets of natural numbers beginning with 1 and 0 , respectively, i.e. $\mathbb{N}_{0}=\mathbb{N} \cup\{0\}$.

In order to solve problem (1.1) - (1.4) by semidiscretization in time (Rothe method) we subdivide the time interval $I$ by points $t_{j}=j h \quad(h>0 ; j=0, \ldots, n)$ and replace $(1.1)-(1.3)$ by the time discretized problem (in weak formulation; $j=1, \ldots, n$ )

$$
\begin{aligned}
\left\langle\delta u_{j}, v\right\rangle+A_{j}\left(u_{j}, v\right) & =\left\langle f_{j}, v\right\rangle & & \text { for all } v \in W_{0}^{1, p^{\prime}}(G) \\
u_{j} & =0 & & \text { on } \partial G \\
u_{0} & =U_{0} & &
\end{aligned}
$$

where

$$
\delta u_{j}=\frac{u_{j}-u_{j-1}}{h}, \quad f_{j}=f\left(x, t_{j}, u_{j-1}\right), \quad A_{j}(\cdot, \cdot)=A_{\left(\imath_{j}, u_{j-1}\right)}(\cdot, \cdot) .
$$

Starting from (2.3) $)_{0}$ thus we have to solve a set of linear elliptic boundary value problems $(2.1)_{j},(2.2)_{j}$ to obtain the approximations $u_{j}$. By interpolation with respect to time this yields the Rothe functions

$$
\tilde{u}^{n}(x, t)=\frac{t_{j}-t}{h} u_{j-1}(x)+\frac{t-t_{j-1}}{h} u_{j}(x) \quad\left(t \in\left[t_{j-1}, t_{j}\right]\right)
$$


and

$$
\bar{u}^{n}(x, t)= \begin{cases}u_{j}(x) & \text { if } t \in\left(t_{j-1}, t_{j}\right] \\ U_{0}(x) & \text { if } t \leq 0 .\end{cases}
$$

For given $U_{0} \in C(\bar{G})$ and $R>0$ we define the set

$$
\mathcal{M}_{R}\left(U_{0}\right)=\left\{(x, t, u) \in \mathbb{R}^{N+2}: x \in G, t \in I,\left|u-U_{0}(x)\right| \leq R\right\}
$$

and the ball

$$
\mathcal{B}_{R}\left(U_{0}\right)=\left\{u \in C(\bar{G}):\left\|u-U_{0}\right\|_{C(\bar{G})} \leq R\right\} .
$$

We will show that the approximations $\bar{u}^{n}$ and $\tilde{u}^{n}$ and hence the solution $u$ of our problem belong to $\mathcal{B}_{R}\left(U_{0}\right)$ for sufficiently small $t$. Therefore, we suppose the following local conditions.

Assumptions. For given $R>0$ let $a_{i k}, a_{i}$ and $f$ be Carathéodory functions defined on $\mathcal{M}_{R}\left(U_{0}\right)$. Then if $r>N$ and $\mu_{i} \leq \nu<\frac{r N}{N-2}(i=1,2,3)$ with $\frac{N r}{r-2}<\mu_{1}, \frac{N r}{2 r-2}<\mu_{2}$ and $\frac{N r}{2 r+N-2}<\mu_{3}$, we suppose the following:

(i) $U_{0} \in W_{0}^{1, r}(G)$ and $A\left(0, U_{0}\right) U_{0} \in L_{r}(G)$.

(ii) $a_{i k}(\cdot, t, u): I \times \mathcal{B}_{R}\left(U_{0}\right) \rightarrow C(\bar{G})$ and $a_{i}(\cdot, t, u): I \times \mathcal{B}_{R}\left(U_{0}\right) \rightarrow L_{\infty}(G)$ are bounded mappings which fulfil the Lipschitz conditions

$$
\begin{array}{r}
\left\|a_{i k}(\cdot, t, u)-a_{i k}\left(\cdot, t^{\prime}, u^{\prime}\right)\right\|_{\mu_{1}} \leq l_{1}\left(\left|t-t^{\prime}\right|+\left\|u-u^{\prime}\right\|_{\nu}\right) \\
\left\|a_{i}(\cdot, t, u)-a_{i}\left(\cdot, t^{\prime}, u^{\prime}\right)\right\|_{\mu_{2}} \leq l_{2}\left(\left|t-t^{\prime}\right|+\left\|u-u^{\prime}\right\|_{\nu}\right)
\end{array}
$$

for all $t, t^{\prime} \in I$ and $u, u^{\prime} \in \mathcal{B}_{R}\left(U_{0}\right)$ as well as the ellipticity condition

$$
\sum_{i, k} a_{i k}(x, t, u) \xi_{i} \xi_{k} \geq a \xi^{2}
$$

for all $(x, t, u) \in \mathcal{M}_{R}\left(U_{0}\right)$ and $\xi \in \mathbb{R}^{N}, a>0$ being some constant.

(iii) $f(\cdot, t, u): I \times \mathcal{B}_{R}\left(U_{0}\right) \rightarrow L_{r}(G)$ is bounded and fulfils the Lipschitz condition

$$
\left\|f(\cdot, t, u)-f\left(\cdot, t^{\prime}, u^{\prime}\right)\right\|_{\mu_{3}} \leq l_{3}\left(\left|t-t^{\prime}\right|+\left\|u-u^{\prime}\right\|_{\nu}\right)
$$

for all $t, t^{\prime} \in I$ and $u, u^{\prime} \in \mathcal{B}_{R}\left(U_{0}\right)$.

Example. We consider the equation

$$
u_{t}-\nabla\left(\frac{1-u^{2}}{|x|+u} \nabla u\right)+b(x, t)(\tan u)_{x_{1}}=\frac{1}{|x|^{\alpha}} e^{u}
$$

in $G=\left\{x \in \mathbb{R}^{N}:|x|<1\right\}$ with homogeneous boundary condition (1.2) and initial function $U_{0}=\frac{1-|x|^{2}}{2}$. The coefficient $b=b(x, t)$ will be defined by

$$
b(x, t)= \begin{cases}\phi\left(\frac{|x|^{p}}{t}\right) & \text { for } t>0 \\ 0 & \text { for } t=0\end{cases}
$$


where $\phi: \mathbb{R}^{+} \rightarrow \mathbb{R}$ is a Lipschitz function with supp $\phi \subset[0,1]$. If $\alpha<1$ and $0<$ $\beta \leq \frac{2(N-\alpha)}{N}$, one can choose $N<r<\frac{N}{\alpha}$ and $\frac{N r}{2 r-2}<\mu_{2}<\frac{N}{\beta}$. Then for $R<\frac{1}{2}$ this problem fulfils the above Assumptions (i) - (iii) if $\nu$ and $\mu_{3}$ are choosen in such way that $\frac{\nu}{\mu_{3}} \geq \frac{r+N-2}{N-2}$.

Note that the coefficient $b=b(x, t)$ in the example does not fulfil any Lipschitz condition with respect to $t$.uniformly in $x \in G$. Moreover, for $\phi(s)=\max \{1-s, 0\}$ the smallest pointwise Lipschitz constant $L=L(x)=\frac{1}{|x|^{\beta}}$ does not belong to $L_{N / \beta}(G)$, however the second Lipschitz condition in Assumption (ii) is fulfilled for $\mu_{2}=\frac{N}{\beta}$.

For a given function $\psi: \mathcal{M}_{R}\left(U_{0}\right) \rightarrow \mathbb{R}$ we define the cut function $\psi^{R}$ by

$$
\psi^{R}(x, t, u)= \begin{cases}\psi(x, t, u) & \text { for }(x, t, u) \in \mathcal{M}_{R}\left(U_{0}\right) \\ \psi\left(x, t, U_{0}(x)+R \operatorname{sign}\left(u-U_{0}(x)\right)\right. & \text { otherwise. }\end{cases}
$$

For the following calculations we replace the coefficients $a_{i k}$ and $a_{i}$ in $A$ (see (1.4)) by $a_{i k}^{R}$ and $a_{i}^{R}$, respectively, and the right-hand side $f$ in (1.1) by $f^{R}$. Obviously, these functions fulfil Assumptions (ii) and (iii) globally for all $u \in C(\bar{G})$ instead of $u \in \mathcal{B}_{R}\left(U_{0}\right)$. In Theorem 3.1 we will prove that the argument $u=u_{j-1}$ belongs to $\mathcal{B}_{R}\left(U_{0}\right)$ for sufficiently small $t \in \hat{I}$, therefore we may identify $a_{i k}^{R}$ and $a_{i}^{R}$ with $a_{i k}$ and $a_{i}$, respectively, and $f^{R}$ with $f$. For simplicity we drop the superscript $R$ from the beginning.

Starting from the given $U_{0}$ in $(2.3)_{0}$ there exist unique solutions $u_{j} \in W_{0}^{1, r}(G)$ of the truncated equations $(2.1)_{j}$ for all $h \leq h_{0}(j=1,2, \ldots, \dot{n})$ (see [11: Corollary 7.4]). Since $r>N$ this implies $u_{j} \in C^{\lambda}(\bar{G})\left(\lambda=1-\frac{N}{r}\right)$ and $\left\|u_{j}\right\|_{\infty}=\left\|u_{j}\right\|_{C(\bar{G})}$.

We list some auxiliary assertions which we need for the estimates.

Lemma 2.1. Let $u, v \in W_{0}^{1, r}(G)(r>N)$ and $u^{\prime}, u^{\prime \prime} \in C(\bar{G})$. Moreover, define $w=|u|^{(p-2) / 2} u$ for $p \geq 2$, and suppose Assumption (ii). Then

$$
|u|^{p-2} u \in W_{0}^{1, r}(G) \quad \text { with } \nabla\left(|u|^{p-2} u\right)=(p-1)|u|^{p-2} \nabla u
$$

and it holds:

$$
\left\||u|^{p-2} u\right\|_{p^{\prime}}=\|u\|_{p}^{p-1} \quad \text { and } \quad\|w\|_{2}^{2}=\|u\|_{p}^{p} \quad\left(\frac{1}{p}+\frac{1}{p^{\prime}}=1\right)
$$

and

$$
\begin{aligned}
& A_{\left(t, u^{\prime}\right)}\left(u,|u|^{p-2} u\right) \geq k_{1}\|w\|_{1,2}^{2}-k_{2}\|u\|_{p}^{p} \\
&\left|A_{\left(t, u^{\prime}\right)}\left(v,|u|^{p-2} u\right)\right| \leq c\|v\|_{1, r}\|w\|_{1,2}\|w\|_{s}^{(p-2) / p} \\
&\left|\left(A_{\left(t^{\prime}, u^{\prime}\right)}-A_{\left(t^{\prime \prime}, u^{\prime \prime}\right)}\right)\left(v ;|u|^{p-2} u\right)\right| \leq c\left(\left|t^{\prime}-t^{\prime \prime}\right|+\left\|u^{\prime}-u^{\prime \prime}\right\|_{\nu}\right) \\
& \times\|v\|_{1, r}\|w\|_{1,2}\|w\|_{s}^{(p-2) / p}
\end{aligned}
$$

with $p \leq r$ in (2.9), $k_{1}=\frac{(2 p-2) a}{p^{2}} \geq \frac{\text { const }}{p}, k_{2}=\frac{\text { const }}{p}$ and $s<\frac{2 N}{N-2}$. 
Proof. The proof that $|u|^{p-2} u$ belongs to $W_{0}^{1, r}(G)$ and that of relations (2.6) and (2.7) is given in [8: Lemmas 2 and 3]. In order to prove relations (2.8) and (2.9) we estimate a bilinear form generated by an elliptic operator $\dot{A}$ with coefficients $\tilde{a}_{i k} \in$ $L_{\lambda_{1}}(G)$ and $\tilde{a}_{i} \in L_{\lambda_{2}}(G)$, where $1 \leq \lambda_{1}, \lambda_{2} \leq+\infty$. By means of

$$
\begin{aligned}
\nabla\left(|u|^{p-2} u\right) & =(p-1)|u|^{p-2} \nabla u \\
& =(p-1)|u|^{(p-2) / 2}\left(|u|^{(p-2) / 2} \nabla u\right) \\
& =2 \frac{p-1}{p}|u|^{(p-2) / 2} \nabla\left(|u|^{(p-2) / 2} u\right) \\
& =2 \frac{p-1}{p}|w|^{(p-2) / p} \nabla w
\end{aligned}
$$

we obtain

$$
\begin{aligned}
\left|\tilde{A}\left(v,|u|^{(p-2)} u\right)\right| & \leq c \max _{i, k} \int_{G}\left|\tilde{a}_{i k}\right||\nabla v|\left|\nabla\left(|u|^{p-2} u\right)\right| d x+c \max _{i} \int_{G}\left|\tilde{a}_{i}\right||\nabla v||u|^{p-1} d x \\
& \leq c \max _{i, k} \int_{G} \underbrace{\left|\tilde{a}_{i k}\right|}_{A_{1}} \underbrace{|\nabla v|}_{A_{2}} \underbrace{|\nabla w|}_{A_{3}} \underbrace{|w|^{(p-2) / p}}_{A_{4}} d x+c \max _{i} \int_{G} \underbrace{\left|\tilde{a}_{i}\right|}_{B_{1}} \underbrace{|\nabla v|}_{B_{2}} \underbrace{|w|^{2(p-1) / p}}_{B_{3}} d x .
\end{aligned}
$$

First let $p>2$. We now apply the Hölder inequality with exponents $\alpha_{i}$ and $\beta_{i}$ to the integrals with factors $A_{i}$ and $B_{i}$, respectively. Especially we choose

$$
\begin{array}{ll}
\alpha_{1}=\lambda_{1}, & \alpha_{2}=r, \quad \alpha_{3}=2, \quad \alpha_{4}=\frac{p s}{p-2} . \\
\beta_{1}=\lambda_{2}, & \beta_{2}=r, \quad \beta_{3}=\frac{p s}{2(p-1)} \text { with } s<\frac{2 N}{N-2} .
\end{array}
$$

Hence

$$
\begin{aligned}
\left|\tilde{A}\left(v,|u|^{p-2} u\right)\right| \leq & c\left(\max _{i, k}\left\|\tilde{a}_{i k}\right\|_{\lambda_{1}}\|\nabla v\|_{r}\|\nabla w\|_{2}\|w\|_{s}^{(p-2) / p}\right. \\
& \left.+\max _{i}\left\|\tilde{a}_{i}\right\|_{\lambda_{2}}\|\nabla v\|_{r}\|w\|_{s}^{(2 p-2) / p}\right) \\
\leq & c\left(\max _{i, k}\left\|\tilde{a}_{i k}\right\|_{\lambda_{1}}+\max _{i}\left\|\tilde{a}_{i}\right\|_{\lambda_{2}}\right)\|v\|_{1, r}\|w\|_{1,2}\|w\|_{s}^{(p-2) / p} .
\end{aligned}
$$

In the last estimate the continuous embedding $W_{0}^{1,2}(G) \subset L_{s}(G)$ was used. In order to ensure $\sum_{i=1}^{4} \alpha_{i}^{-1}=1$ and $\sum_{i=1}^{3} \beta_{i}^{-1}=1$ the Lebesgue exponents $\lambda_{1}$ and $\lambda_{2}$ in $(2.10)$ have to fulfil the conditions

$$
\lambda_{1}>\frac{p r N}{p(r-N)+r(N-2)} \quad \text { and } \quad \lambda_{2}>\frac{p r N}{p(2 r-N)+r(N-2)}
$$

Since the right-hand sides in (2.11) are bounded from above for all $p \geq 2$ and $\tilde{a}_{i k}=$ $a_{i k}\left(\cdot, t, u^{\prime}\right) \in L_{\infty}(G), \tilde{a}_{i}=a_{i}\left(\cdot, t, u^{\prime}\right) \in L_{\infty}(G)$ this yields relation (2.8).

To prove relation $(2.9)$ we choose $\tilde{a}_{i k}=a_{i k}\left(\cdot, t^{\prime}, u^{\prime}\right)-a_{i k}\left(\cdot, t^{\prime \prime}, u^{\prime \prime}\right), \tilde{a}_{i}$ analogously, and $\lambda_{1}=\mu_{1}, \lambda_{2}=\mu_{2}$. Then (2.11) is fulfilled for $2<p \leq r$ in view of the restrictions on $\mu_{i}$. Estimation of (2.10) by means of the Lipschitz conditions (ii) yields (2.9):

Finally, if $p=2$, then the term $A_{4}$ disappears. Hence we set $\alpha_{4}=0$ and have $\alpha_{1}=\frac{2 r}{r-2}<\mu_{1}, \alpha_{2}=r$ and $\alpha_{3}=2$ :.Obviously, the assertion holds, too 
An essential tool in our investigations is the Nirenberg-Gagliardo interpolation inequality (see [6: pp. $80-84]$ ). Let $1 \leq q \leq p \leq s$ and $\frac{1}{p}<\frac{1}{s}+\frac{1}{N}$. Then for all $u \in W_{0}^{1, p}(G)$ we have

$$
\|u\|_{0} \leq c_{1}\|u\|_{1, p}^{\theta}\|u\|_{q}^{1-\theta} \quad \text { with } \quad \bar{\theta}=\frac{\frac{1}{q}-\frac{1}{s}}{\frac{1}{q}-\frac{1}{p}+\frac{1}{N}} \leq \theta \leq 1 .
$$

If $q=1$, then $\bar{\theta}<\theta \leq 1$. As a corollary of this inequality we get

Lemma 2.2. Let $2 \leq s<\frac{2 N}{N-2}$ and $u \in W_{0}^{1,2}(G)$. Then for $\varepsilon>0, \alpha>0$ and $1 \leq q \leq 2$ there holds

$$
\|u\|_{s}^{2 \alpha} \leq \varepsilon\|u\|_{1,2}^{2}+c_{e}\|u\|_{q}^{2 \beta}
$$

where we distinguish the following cases:

a) If $0<\alpha<1$, then $0<\beta \leq \bar{\beta}<\alpha$ and $c_{e} \sim \varepsilon^{-\frac{\alpha-\beta}{1-\alpha}}$.

b) If $\alpha=1$, then $\beta=1$ and $c_{c} \sim \varepsilon^{-\sigma}$ with $\bar{\sigma} \leq \sigma<+\infty$.

c) If $1<\alpha<\bar{\alpha}$, then $\alpha<\bar{\beta} \leq \beta<+\infty$ and $c_{\varepsilon} \sim \varepsilon^{-\frac{\beta-\alpha}{\alpha-1}}$

with

$$
\bar{\alpha}=\frac{1+\bar{\sigma}}{\bar{\sigma}}, \quad \bar{\beta}=\frac{\alpha}{1+(1-\alpha) \bar{\sigma}}, \quad \bar{\sigma}=\frac{2 N(s-q)}{q[2 N-(N-2) s]} .
$$

If $q=1$, then the choice $\beta=\bar{\beta}$ and $\sigma=\bar{\sigma}$ is excluded.

Proof. We start with (2.12) for $p=2$ and obtain for $\alpha \neq 1$ by means of the Young inequality

$$
\|u\|_{s}^{2 \alpha} \leq c_{1}^{2 \alpha}\|u\|_{1,2}^{2 \alpha \theta}\|u\|_{q}^{2 \alpha(1-\theta)} \leq \varepsilon\|u\|_{1,2}^{2}+c_{\varepsilon}\|u\|_{q}^{2 \frac{\alpha-\alpha \theta}{1-\alpha \theta}}
$$

provided that $\alpha \theta<1$. This yields the condition

$$
\alpha<\bar{\alpha}:=\frac{1}{\bar{\theta}} \quad \text { with } \quad \bar{\theta}=\frac{2 N(s-q)}{s[2 N-(N-2) q]}
$$

from (2.12), which is restrictive only in case c). Moreover, $c_{\varepsilon} \sim \varepsilon^{-\alpha \theta /(1-\alpha \theta)}$. Defining $\beta=\beta(\theta):=\frac{\alpha-\alpha \theta}{1-\alpha \theta}$ we obtain $\frac{\alpha \theta}{1-\alpha \theta}=\frac{\alpha-\beta}{1-\alpha}$. Since $\theta$ may be chosen within the interval $[\bar{\theta}, 1)$ we investigate the range of $\beta$ on $[\bar{\theta}, 1)$ using the derivative $\beta^{\prime}(\theta)=\frac{\alpha(\alpha-1)}{(1-\alpha \theta)^{2}}$. In case a) the function $\beta=\beta(\theta)$ is monotonically degreasing on $[0,1]$ with $\beta(0)=\alpha$ and $\beta(1)=0$. Hence, $0<\beta \leq \bar{\beta}$ with $\bar{\beta}=\beta(\bar{\theta})$ and $\bar{\beta}<\alpha$. In case c) the function $\beta=\beta(\theta)$ is monotonically increasing on $\left[0, \frac{1}{\alpha}\right)$ with $\beta(0)=\alpha$ and a pole in $\theta=\frac{1}{\alpha}$. Hence, $\bar{\beta} \leq \beta<+\infty$ with $\bar{\beta}=\beta(\bar{\theta})$ and now $\bar{\beta}>\alpha$.

It remains to regard the case $\alpha=1$. Applying the Young inequality to (2.12) it follows that $\beta=1$ and $c_{\varepsilon} \sim \varepsilon^{-\theta /(1-\theta)}=\varepsilon^{-\sigma}$. Varying $\theta$ in $[\bar{\theta}, 1)$ we get $\bar{\sigma} \leq \sigma<\infty$ with $\bar{\sigma}=\frac{\bar{\theta}}{1-\bar{\theta}}$.

If $s=q=2$, then $\bar{\theta}=0$. However, the validity of formula (2.13) is obvious in that case even for the choice $\beta=\bar{\beta}=\alpha$ and $\sigma=\bar{\sigma}=0$ 
Remark. If we choose $\beta=\bar{\beta}$, then the exponent $\frac{\alpha-\beta}{1-\alpha}=\frac{\alpha \bar{\sigma}}{1+(1-\alpha) \bar{\sigma}}$ in $c_{\varepsilon}$ tends to $\bar{\sigma}$ for $\alpha \rightarrow 1$.

For estimation of the discrete time derivative to the discretized quasilinear problem we need a nonlinear Gronwall inequality (see Willett and Wong [12]) instead of the well-known linear one, which is used in the linear case. We use the following discrete version.

Lemma 2.3. Let $d_{i}(i=0,1,2, \ldots, n)$ be non-negative real numbers, $K_{0}>0$ and $c_{1}, c_{2}, h, \beta \geq 0$ constants with $\beta \neq 1$. Then the inequality

$$
d_{i} \leq K_{0}+c_{1} \sum_{j=0}^{i-1} h d_{j}+c_{2} \sum_{j=0}^{i-1} h d_{j}^{\beta} \quad(i=0,1, \ldots, n)
$$

implies that

$$
e_{i} d_{i} \leq\left(K_{0}^{1-\beta}+(1-\beta) c_{2} \sum_{j=1}^{i} h e_{j}^{1-\beta}\right)^{1 /(1-\beta)} \quad\left(i=0,1, \ldots, n^{*}\right)
$$

with $e_{i}=\left(1+c_{1} h\right)^{-i}$. Here one has to choose $n^{*} \leq n$ in such way that the condition $(1-\beta) c_{2} \sum_{j=1}^{i} h e_{j}^{1-\beta}<K_{0}^{1-\beta}$ is not violated.

Proof. The assertion of the lemma is a specialization of Theorem 4 in [12] with $u(i+1)=d_{i}(i=0, \ldots, n), v(i)=c_{1} h$ and $w(i)=c_{2} h \quad(i=1, \ldots, n)$

Remark. If $h \leq h_{0}<\frac{1}{c_{1}}$, then the sum $\sum_{j=0}^{i-1} h d_{j}$ may be replaced by $\sum_{j=0}^{i} h d_{j}$ where the assertion holds with $\frac{K_{0}}{1-c_{1} h_{0}}$ and $\frac{c_{k}}{1-c_{1} h_{0}}$ instead of $K_{0}$ and $c_{k} \quad(k=1,2)$, respectively.

Since $i h=t_{i}$ and $1 \geq\left(1+c_{1} h\right)^{-i} \geq e^{-c_{1} i h}=e^{-c_{1} t_{i}}$ we have the following corollary.

Corollary. Suppose the assumtions of Lemma 2.3 with $\beta>1$. Then

$$
d_{i} \leq\left(K_{0}^{-(\beta-1)}-(\beta-1) c_{2} t_{i} e^{c_{1}(\beta-1) t_{i}}\right)^{-1 /(\beta-1)} e^{c_{1} t_{i}}
$$

holds for all $t_{i}$ with $0 \leq t_{i}<t^{*}$ where $t^{*}>0$ is determined as the solution of the equation $(\beta-1) c_{2} t e^{c_{1}(\beta-1) t}=K_{0}^{-(\beta-1)}$. 


\section{A priori estimates for the discretized problem}

We start with $L_{\infty}$-estimates for the solutions $u_{j}$ of the discretized problem (2.1)- (2.3) based on $L_{p}$-estimates followed by a limit process $p \rightarrow+\infty$. For positive constants $\gamma_{1}$ and $\gamma_{2}$ we define

$$
Q_{\gamma_{1}, \gamma_{2}}(\tau)= \begin{cases}\tau^{\gamma_{1}} & \text { if } 0 \leq \tau \leq 1 \\ \tau^{\gamma_{2}} & \text { if } \tau \geq 1\end{cases}
$$

The first lemma yields some tool for performing the limit process.

Lemma 3.1. Let $\left\{m_{\nu}\right\}_{\nu \in N_{0}},\left\{\beta_{\nu}\right\}_{\nu \in N}$ and $\left\{p_{\nu}\right\}_{\nu \in N_{0}}$ be sequences of non-negative real numbers with

$$
0<\beta_{\nu} \leq 1, \quad \prod_{\nu=1}^{\infty} \beta_{\nu}=\beta>0, \quad p_{\nu}=p_{0} \lambda^{\nu} \quad(\lambda>1)
$$

satisfying the recurrence

$$
m_{\nu} \leq\left(c_{1} p_{\nu}^{c_{2}} \tau\left(m_{\nu-1}^{p_{\nu}}+m_{\nu-1}^{p_{\nu} \beta_{\nu}}\right)\right)^{1 / p_{\nu}} \quad(\nu \in \mathbb{N})
$$

for $0 \leq \tau \leq T$ where $c_{1}$ and $c_{2}$ are some positive constants. Then

$$
m_{\infty}:=\limsup _{\nu \rightarrow \infty} m_{\nu} \leq c Q_{\gamma_{1}, \gamma_{2}}(\tau) m_{0}^{\bar{\beta}}
$$

where

$$
\tilde{\beta}=\prod_{\nu=1}^{\infty} \tilde{\beta}_{\nu} \text { with } \tilde{\beta}_{\nu}=\left\{\begin{array}{ll}
\beta_{\nu} & \text { if } m_{\nu}<1 \\
1 & \text { if } m_{\nu} \geq 1
\end{array}, \quad \gamma_{2}=\frac{1}{p_{0}(\lambda-1)}, \quad \gamma_{1}=\beta \gamma_{2} .\right.
$$

Proof. Applying the definition of $\tilde{\beta}_{\nu}$ we estimate

$$
\begin{aligned}
& m_{\nu} \leq\left(c_{1} p_{\nu}^{c_{2}} \tau\right)^{1 / p_{\nu}} \cdot m_{\nu-1}^{\bar{\beta}_{\nu}} \\
& \leq \prod_{i=1}^{\nu}\left(c_{1} p_{i}^{c_{2}} \tau\right)^{\bar{\beta}_{\nu} \cdots \bar{\beta}_{i+1} / p_{i}} \cdot m_{0}^{\bar{\beta}_{2} \cdots \bar{\beta}_{1}} \\
& \leq \prod_{i=1}^{\nu}\left(c_{1} p_{i}^{c_{2}}\right)^{1 / p_{i}} \cdot \tau\left(\sum_{i=1}^{\nu} \bar{\beta}_{\nu} \ldots \bar{\beta}_{i+1} / p_{i}\right) \cdot m_{0}^{\bar{\beta}_{\nu} \ldots \bar{\beta}_{1}} .
\end{aligned}
$$

The first product converges since

$$
\prod_{i=1}^{\nu} p_{i}^{c_{2} / p_{i}}=\prod_{i=1}^{\nu} p_{0}^{c_{2} /\left(p_{0} \lambda^{i}\right)} \lambda^{c_{2} i /\left(p_{0} \lambda^{i}\right)}=p_{0}^{\left(c_{2} / p_{0} \sum_{i=1}^{\nu} 1 / \lambda^{i}\right)} \lambda_{\lambda}^{\left(c_{2} / p_{0} \sum_{i=1}^{\nu} i / \lambda^{i}\right)}
$$

is bounded. The exponent of $\tau$ may be estimated by

$$
\beta \sum_{i=1}^{\nu} \frac{1}{p_{i}} \leq \sum_{i=1}^{\nu} \frac{1}{p_{i}} \prod_{j=i+1}^{\nu} \tilde{\beta}_{j} \leq \sum_{i=1}^{\infty} \frac{1}{p_{i}}=: \gamma_{2} .
$$

Finally, $\prod_{\nu=1}^{\infty} \tilde{\beta}_{j}=\tilde{\beta}>0$ is convergent because of $\beta_{j} \leq \tilde{\beta}_{j} \leq 1$. Passing to the limit $\nu \rightarrow+\infty$ in (3.1) this yields the assertion 
For doing estimations in the next lemma remember that $a_{i k}, a_{i}$ and $f$ at the moment mean the truncated functions which fulfil Assumptions (ii) and (iii) globally.

Lemma 3.2. Let $k \in\{0,1, \ldots, n\}$ be fixed and suppose $\left\|u_{k}\right\|_{1, r} \leq C$ independent of the subdivision for this solution $u_{k}$ of problem $(2.1)_{k},(2.2)_{k}$. Then there are numbers $\lambda_{1}>0$ and $\lambda_{2}>0$ such that the estimate

$$
\left\|u_{j}-u_{k}\right\|_{C(\bar{G})} \leq c Q_{\lambda_{1}, \lambda_{2}}\left(t_{j}-t_{k}\right) \quad \text { for all } t_{j} \in\left[t_{k}, T\right]
$$

holds.

Proof. We define $z_{j}=u_{j}-u_{k}$ for $k \leq j \leq n$. Then $z_{j} \in W_{0}^{1, r}(G)$ fulfils

$$
\left\langle\delta z_{j}, v\right\rangle+A_{j}\left(z_{j}, v\right)=\left\langle f_{j}, v\right\rangle-A_{j}\left(u_{k}, v\right) \quad \text { for all } v \in W_{0}^{1, r^{\prime}}(G)
$$

for $j=k+1, \ldots, n$ with $z_{k}=0$. We insert the test function $v=\left|z_{j}\right|^{p-2} z_{j}$ for $p \geq r$, use the abbreviation $w_{j}=\left|z_{j}\right|^{(p-2) / 2} z_{j}$ and obtain by means of Lemma 2.1

$$
\begin{aligned}
\left\|z_{j}\right\|_{p}^{p}- & \left\|z_{j-1}\right\|_{p}\left\|z_{j}\right\|_{p}^{p-1}+k_{1} h\left\|w_{j}\right\|_{1,2}^{2} \\
& \leq k_{2} h\left\|z_{j}\right\|_{p}^{p}+h\left\|f_{j}\right\|_{r}\left\|z_{j}\right\|_{r^{\prime}(p-1)}^{p-1}+h\left|A_{j}\left(u_{k},\left|z_{j}\right|^{p-2} z_{j}\right)\right|
\end{aligned}
$$

which yields with the Young inequality applied to the second term, with $k_{1}, k_{2}=O\left(\frac{1}{p}\right)$, $\|z ;\|_{p}^{p}=\left\|w_{j}\right\|_{2}^{2}$ and $\left\|f_{j}\right\|_{r} \leq c$ the estimate

$$
\begin{aligned}
& \left\|w_{j}\right\|_{2}^{2}-\left\|w_{j-1}\right\|_{2}^{2}+c h\left\|w_{j}\right\|_{1,2}^{2} \\
& \quad \leq c h\left\|w_{j}\right\|_{2}^{2}+c p h\left\|w_{j}\right\|_{2 r^{\prime}(p-1) / p}^{2(p-1) / p}+p h\left|A_{j}\left(u_{k},\left|z_{j}\right|^{p-2} z_{j}\right)\right|
\end{aligned}
$$

We estimate the last term on the right-hand side of (3.2). Application of the Young inequality to formula (2.8) of Lemma 2.1 yields because of the assumption $\left\|u_{k}\right\|_{1, r} \leq C$

$$
\left|A_{j}\left(u_{k},\left|z_{j}\right|^{p-2} z_{j}\right)\right| \leq \varepsilon\left\|w_{j}\right\|_{1,2}^{2}+\frac{c}{\varepsilon}\left\|w_{j}\right\|_{j}^{2(p-2) / p} .
$$

Let now

$$
\beta(p)=\bar{\beta}=\frac{\alpha}{1+(1-\alpha) \bar{\sigma}}=\frac{p-2}{p+2 \bar{\sigma}}
$$

be the exponent $\bar{\beta}$ corresponding to $\alpha=\frac{p-2}{p}$ idefined in Lemma 2.2. Applying case a) of this lemma with $\tilde{\varepsilon}=\frac{\varepsilon^{2}}{c}$ to the last term of (3.3) we obtain

$$
\left|A_{j}\left(u_{k},\left|z_{j}\right|^{p-2} z_{j}\right)\right| \leq \varepsilon\left\|w_{j}\right\|_{1,2}^{2}+c_{\varepsilon}\left\|w_{j}\right\|_{q}^{2 \beta(p)} \quad\left(c_{\varepsilon} \sim \varepsilon^{-\sigma}\right)
$$

with $1<q \leq 2$. Observe that

$$
\sigma=\sigma(p)=2 \frac{\alpha-\beta}{1-\alpha}+1=\frac{p(2 \bar{\sigma}+1)-2 \bar{\sigma}}{p+2 \bar{\sigma}} \leq \sigma_{M}
$$


remains bounded as $p \rightarrow+\infty$.

Since the first term on the right-hand side of (3.2) can be estimated in the same way by Lemma $2.2 / \mathrm{b}$ ) and the second one with the help of the continouos embedding $W_{0}^{1,2}(G) \subset L_{2 r /(r-2)}(G)$ by

$$
\left\|w_{j}\right\|_{2 r^{\prime}(p-1) / p}^{2(p-1) / p} \leq c\left\|w_{j}\right\|_{2 r /(r-2)}^{2(p-1) / p} \leq c\left\|w_{j}\right\|_{1,2}\left\|w_{j}\right\|_{2 r /(r-2)}^{(p-2) / p}
$$

leading to the same term as in (2.8), we may continue to estimate (3.2) by

$$
\left\|w_{j}\right\|_{2}^{2}-\left\|w_{j-1}\right\|_{2}^{2} \leq c h\left\|w_{j}\right\|_{q}^{2}+c h p^{\sigma_{M}+1}\left\|w_{j}\right\|_{q}^{2 \beta(p)}
$$

where $\varepsilon=\frac{\delta}{p}$ with small $\delta>0$ was fixed. Summing up these inequalities for $k+1 \leq j \leq i$ and rewriting into terms of $z_{j}=u_{j}-u_{k}(k \leq j \leq n)$ we obtain

$$
\begin{aligned}
\left\|z_{i}\right\|_{p}^{p} & \leq c h p^{c} \sum_{j=k+1}^{i}\left(\left\|z_{j}\right\|_{p q / 2}^{p}+\left\|z_{j}\right\|_{p q / 2}^{p \beta(p)}\right) \\
& \leq c p^{c}\left(t_{i}-t_{k}\right)\left(\max _{k \leq j \leq i}\left\|z_{j}\right\|_{p q / 2}^{p}+\max _{k \leq j \leq i}\left\|z_{j}\right\|_{p q / 2}^{p \beta(p)}\right)
\end{aligned}
$$

hence

$$
\max _{k \leq j \leq i}\left\|z_{j}\right\|_{p}^{p} \leq c p^{c}\left(t_{i}-t_{k}\right)\left(\max _{k \leq j \leq i}\left\|z_{j}\right\|_{p q / 2}^{p}+\max _{k \leq j \leq i}\left\|z_{j}\right\|_{p q / 2}^{p \beta(p)}\right)
$$

for every $p \geq r$. In order to estimate the limit $\lim _{p \rightarrow \infty}\left\|z_{j}\right\|_{p}=\left\|z_{j}\right\|_{\infty}$ we fix $q \in(1,2)$ and choose the special sequence $p_{\nu}=r\left(\frac{2}{q}\right)^{\nu} \quad\left(\nu \in \mathbb{N}_{0}\right)$. Defining

$$
m_{\nu}=\max _{k \leq j \leq i}\left\|z_{j}\right\|_{p_{\nu}} \quad \text { and } \quad \beta_{\nu}=\beta\left(p_{\nu}\right)
$$

we get the recurrence

$$
m_{\nu} \leq\left(c p_{\nu}^{c}\left(t_{i}-t_{k}\right)\left(m_{\nu-1}^{p_{\nu}}+m_{\nu-1}^{p_{\nu} \beta_{\nu}}\right)\right)^{1 / p_{\nu}} \quad(\nu \in \mathbb{N}) .
$$

In order to apply Lemma 3.1 we state by means of $(3.4)$ that

$$
\prod_{i=1}^{\infty} \beta_{i}=\prod_{i=1}^{\infty}\left(1-\frac{2(\bar{\sigma}+1)}{p_{i}+2 \bar{\sigma}}\right)
$$

is convergent since

$$
\sum_{i=1}^{\infty} \frac{2(\bar{\sigma}+1)}{p_{i}+2 \bar{\sigma}} \leq 2(\bar{\sigma}+1) \sum_{i=1}^{\infty} \frac{1}{p_{i}}
$$

converges. Hence, by Lemma 3.1,

$$
m_{\infty} \leq c Q_{\gamma_{1}, \gamma_{2}}\left(t_{i}-t_{k}\right) m_{0}^{\bar{\beta}} \quad \text { with } \quad 0<\beta \leq \tilde{\beta} \leq 1
$$


It remains to estimate $m_{0}=\max _{k \leq j \leq i}\left\|z_{j}\right\|_{r}$. To do this we start from (3.5) with $p=r$ and $q=2$, and obtain due to $a^{\beta} \leq 1+a$

$$
\left\|z_{i}\right\|_{r}^{r} \leq c h(i-k)+c h \sum_{j=k+1}^{i}\left\|z_{j}\right\|_{r}^{r} .
$$

The discrete linear Gronwall lemma (Lemma 2.3 with $c_{2}=0$ ) yields for $h \leq h_{0}$

$$
\left\|z_{j}\right\|_{r}^{r} \leq c h(j-k) e^{c h(j-k)}=c\left(t_{j}-t_{k}\right) e^{c\left(t_{j}-t_{k}\right)}
$$

hence $m_{0} \leq c\left(t_{i}-t_{k}\right)^{1 / r} e^{c\left(t_{i}-t_{k}\right)}$. Finally, inserting this into (3.6) we get

$$
\max _{k \leq j \leq i}\left\|z_{j}\right\|_{\infty} \leq c Q_{\gamma_{1}, \gamma_{2}}\left(t_{i}-t_{k}\right)\left(t_{i}-t_{k}\right)^{\tilde{\beta} / r} e^{c\left(t_{i}-t_{k}\right)}
$$

Since $\tilde{\beta}$ depends on the subdivision we replace it by $\beta$ if $\left(t_{i}-t_{k}\right)<1$, and by 1 else. This completes the proof with $\lambda_{1}=\gamma_{1}+\frac{\beta}{r}=\frac{2 \beta}{r(2-q)}$ and $\lambda_{2}=\gamma_{2}+\frac{1}{r}=\frac{2}{r(2-q)}$

A simple conclusion of Lemma 3.2 is the local boundedness of the approximations.

Theorem 3.1. Suppose Assumptions (i) - (iii) with some $R>0$. Then there is a time $\widehat{T}$ with $0<\widehat{T} \leq T$, independent of the subdivision such that the solutions $u_{j}$ of problems $(2.1)_{j},(2.2)_{j}$ belong to $\mathcal{B}_{R}\left(U_{0}\right)$ for all $t_{j} \in \hat{I}=[0, \hat{T}]$.

Proof. We choose $k=0$ in Lemma 3.2 and obtain due to $\left\|U_{0}\right\|_{1, r}=C$

$$
\left\|u_{j}-U_{0}\right\|_{C(\bar{G})} \leq c Q_{\lambda_{1}, \lambda_{2}}\left(t_{j}\right)
$$

Since $Q_{\lambda_{1}, \lambda_{2}}(0)=0$ there is a $\hat{t}>0$ such that $c Q_{\lambda_{1}, \lambda_{2}}(t) \leq R$ for all $t \leq \hat{t}$. Then $\widehat{T}=\min \{\hat{t}, T\}$

The assertion of Theorem 3.1 means that the solutions $u_{j} \in W_{0}^{1, r}(G)$ of the truncated problem are solutions of the non-truncated original equations $(2.1)_{j}$ for all $t_{j} \leq \widehat{T}$. From now we only regard this interval $\hat{I}=[0, \widehat{T}]$.

Theorem 3.1 especially implies

$$
\left\|u_{j}\right\|_{\infty} \leq C_{1} \quad \text { and } \quad\left\|f_{j}\right\|_{r} \leq c \quad \text { for all } t_{j} \in \hat{I} .
$$

Since $u_{j} \in W_{0}^{1, r}(G)$ fulfils the elliptic equation $A_{j} u_{j}=F_{j}$ with $F_{j}=f_{j}-\delta u_{j}$ we can use an a priori estimate for weak solutions of elliptic Dirichlet problems (see Simader [11: Theorem 6.3]) and obtain by means of (3.7)

$$
\left\|u_{j}\right\|_{1, r} \leq c_{1}\left\|F_{j}\right\|_{r}+c_{2}\left\|u_{j}\right\|_{r} \leq c\left(1+\left\|\delta u_{j}\right\|_{r}\right) \quad \text { for all } t_{j} \in \hat{I} .
$$

This inequality is applied in the next lemma that yields boundedness of the discrete time derivative. 
Lemma 3.3. Suppose Assumptions (i) - (iii). Then for $h \leq h_{0}$ there is a time interval $\left[0, T^{*}\right] \subset[0, \hat{T}]$ such that the estimate.

$$
\left\|\delta u_{j}\right\|_{r} \leq C_{2} \quad \text { for all } t_{j} \in\left[0, T^{*}\right]
$$

holds independently of the subdivision.

Proof. We take the difference $(2.1)_{j}-(2.1)_{j-1}(j=2, \cdots, \hat{n})$ and testing it with $v=\left|\delta u_{j}\right|^{r-2} \delta u_{j}$ we get

$$
\begin{aligned}
\left\langle\delta u_{j}\right. & \left.-\delta u_{j-1},\left|\delta u_{j}\right|^{r-2} \delta u_{j}\right\rangle+h A_{j}\left(\delta u_{j},\left|\delta u_{j}\right|^{r-2} \delta u_{j}\right) \\
& =-\left(A_{j}-A_{j-1}\right)\left(u_{j-1},\left|\delta u_{j}\right|^{r-2} \delta u_{j}\right)+\left\langle f_{j}-f_{j-1},\left|\delta u_{j}\right|^{r-2} \delta u_{j}\right\rangle .
\end{aligned}
$$

This relation may be estimated by means of Lemma 2.1 and Assumption (iii), where $\omega_{j}=\left|\delta u_{j}\right|^{(r-2) / 2} \delta u_{j}$. Hence

$$
\begin{aligned}
& \left\|\delta u_{j}\right\|_{r}^{r}-\left\|\delta u_{j-1}\right\|_{r}\left\|\delta u_{j}\right\|_{r}^{r-1}+k_{1} h\left\|\omega_{j}\right\|_{1,2}^{2} \\
& \leq \\
& \quad k_{2} h\left\|\delta u_{j}\right\|_{r}^{r}+c h\left(1+\left\|\delta u_{j-1}\right\|_{\nu}\right)\left\|u_{j-1}\right\|_{1, r}\left\|\omega_{j}\right\|_{1,2}\left\|\omega_{j}\right\|_{s}^{(r-2) / r} \\
& \quad+l_{3} h\left(1+\left\|\delta u_{j-1}\right\|_{\nu}\right)\left\|\delta u_{j}\right\|_{\mu_{3}^{\prime}(r-1)}^{r-1}
\end{aligned}
$$

From (3.8) and the Hölder inequality there follows

$$
\begin{aligned}
&\left\|\delta u_{j}\right\|_{r}^{r}-\left\|\delta u_{j-1}\right\|_{r}^{r}+k_{1} h r\left\|\omega_{j}\right\|_{1,2}^{2} \\
& \leq c h\left(1+\left\|\delta u_{j}\right\|_{r}^{r}+\left\|\delta u_{j}\right\|_{\mu_{3}^{\prime}(r-1)}^{r}+\left\|\delta u_{j-1}\right\|_{\nu}^{r}\right. \\
&+\underbrace{\left.\left(1+\left\|\delta u_{j-1}\right\|_{\nu}\right)\left(1+\left\|\delta u_{j-1}\right\|_{r}\right)\left\|\omega_{j}\right\|_{1,2}\left\|\omega_{j}\right\|_{s}^{(r-2) / r}\right)}_{(*)} .
\end{aligned}
$$

We estimate the last line $(*)$ of (3.10) separately and get

$$
\begin{aligned}
\left(^{*}\right) \leq & \left(1+\left\|\delta u_{j-1}\right\|_{r}\right)\left\|\omega_{j}\right\|_{1,2}\left\|\omega_{j}\right\|_{s}^{(r-2) / r} \\
& +\left\|\delta u_{j-1}\right\| \nu\left\|\omega_{j}\right\|_{1,2}\left\|\omega_{j}\right\|_{s}^{(r-2) / r} \\
& +\left\|\delta u_{j-1}\right\|_{\nu}\left\|\delta u_{j-1}\right\|_{r}\left\|\omega_{j}\right\|_{1,2}\left\|\omega_{j}\right\|_{s}^{(r-2) / r}
\end{aligned}
$$

Further, applying the Young inequality with exponents $p_{1}=r, p_{2}=2$ and $p_{3}=\frac{2 r}{r-2}$ to the first two items and with exponents $p_{1}>r, p_{2}>r, p_{3}=2$ and $p_{4}=\frac{2 r}{r-2} \quad\left(\sum \frac{1}{p_{i}}=1\right)$ to the last one we get

$$
\begin{aligned}
(*) \leq & \varepsilon\left\|\omega_{j}\right\|_{1,2}^{2} \\
& +c\left(1+\left\|\ddot{\omega}_{j}\right\|_{j}^{2}+\left\|\delta u_{j-1}\right\|_{r}^{r}+\left\|\delta u_{j-1}\right\|_{\nu}^{r}+\left\|\delta u_{j-1}\right\|_{\nu}^{p_{1}}+\left\|\delta u_{j-1}\right\|_{r}^{p_{2}}\right)
\end{aligned}
$$


Now if we rewrite $\left|\delta u_{j}\right|=\left|\omega_{j}\right|^{2 / r}$, then

$$
\left\|\delta u_{j-1}\right\|_{\nu}^{r}=\left\|\omega_{j-1}\right\|_{2 \nu / r}^{2}, \quad\left\|\delta u_{j}\right\|_{\mu_{3}^{\prime}(r-1)}^{r}=\left\|\omega_{j}\right\|_{2 \mu_{3}^{\prime}(r-1) / r}^{2}, \quad\left\|\delta u_{j}\right\|_{r}^{r}=\left\|\omega_{j}\right\|_{2}^{2}
$$

where $\frac{2 \nu}{r}=s_{1}$ and $\frac{2 \mu_{3}^{\prime}(r-1)}{r}=s_{2}$ are less than $\frac{2 N}{N-2}$ because of the conditions on $\nu$ and $\mu_{3}$, respectively. Thus we obtain from (3.10) for small fixed $\varepsilon>0$

$$
\begin{aligned}
& \left\|\omega_{j}\right\|_{2}^{2}-\left\|\omega_{j-1}\right\|_{2}^{2}+c h\left\|\omega_{j}\right\|_{1,2}^{2} \\
& \quad \leq \operatorname{ch}\left(1+\left\|\omega_{j}\right\|_{2}^{2}+\left\|\omega_{j}\right\|_{s}^{2}+\left\|\omega_{j-1}\right\|_{s}^{2}+\left\|\omega_{j-1}\right\|_{s}^{2 p_{1} / r}+\left\|\omega_{j-1}\right\|_{2}^{2 p_{2} / r}\right) .
\end{aligned}
$$

Depending on $s<\frac{2 N}{N-2}$ we fix now $\alpha_{1}=\frac{p_{1}}{r}$ such that the conditions of Lemma 2.2/c) are fulfilled with $q=2$. Then $\alpha_{2}=\frac{p_{2}}{r}>1$ is also fixed. Application of this lemma with $q=2$ to the items $\left\|\omega_{j}\right\|_{s}^{2},\left\|\omega_{j-1}\right\|_{s}^{2}$ and $\left\|\omega_{j-1}\right\|_{s}^{2 p_{1} / r}$ yields

$$
\begin{aligned}
& \left\|\omega_{j}\right\|_{2}^{2}-\left\|\omega_{j-1}\right\|_{2}^{2}+c h\left\|\omega_{j}\right\|_{1,2}^{2} \\
& \quad \leq \varepsilon h\left(\left\|\omega_{j}\right\|_{1,2}^{2}+\left\|\omega_{j-1}\right\|_{1,2}^{2}\right)+c_{\varepsilon} h\left(1+\left\|\omega_{j}\right\|_{2}^{2}+\left\|\omega_{j-1}\right\|_{2}^{2}+\left\|\omega_{j-1}\right\|_{2}^{2 \beta}\right)
\end{aligned}
$$

with $\beta=\max \left\{\bar{\beta}_{1}, \alpha_{2}\right\}>1$. Summing up these inequalities for $j=2, \ldots, i$ we obtain for sufficiently small $\varepsilon$

$$
\begin{aligned}
&\left\|\omega_{i}\right\|_{2}^{2}+c h \sum_{j=2}^{i}\left\|\omega_{j}\right\|_{1,2}^{2} \\
& \leq\left\|\omega_{1}\right\|_{2}^{2}+c\left(t_{i}+h\left\|\omega_{1}\right\|_{1,2}^{2}+\sum_{j=1}^{i} h\left\|\omega_{j}\right\|_{2}^{2}+\sum_{j=1}^{i-1} h\left\|\omega_{j}\right\|_{2}^{2 \beta}\right)
\end{aligned}
$$

for $i=2, \ldots, \hat{n}$.

In order to apply Lemma 2.3 it remains to estimate $\left\|\omega_{1}\right\|_{2}^{2}+h\left\|\omega_{1}\right\|_{1,2}^{2}$. To do this we insert $v=\left|\delta u_{1}\right|^{r-2} \delta u_{1}$ into relation (2.1) 1 getting

$$
\left\|\delta u_{1}\right\|_{r}^{r}+h A_{1}\left(\delta u_{1},\left|\delta u_{1}\right|^{r-2} \delta u_{1}\right)=\left\langle f_{1},\left|\delta u_{1}\right|^{r-2} \delta u_{1}\right\rangle-A_{1}\left(U_{0},\left|\delta u_{1}\right|^{r-2} \delta u_{1}\right)
$$

and obtain by means of (2.7), Assumptions (i) and (ii), and (2.9)

$$
\begin{aligned}
\left\|\delta u_{1}\right\|_{r}^{r} & +k_{1} h\left\|\omega_{1}\right\|_{1,2}^{2} \\
\leq & \left\|f_{0}-A\left(0, U_{0}\right) U_{0}\right\|_{r}\left\|\delta u_{1}\right\|_{r}^{r-1}+\left\|f_{1}-f_{0}\right\|_{\mu_{3}}\left\|\delta u_{1}\right\|_{\mu_{3}^{\prime}(r-1)}^{r-1} \\
& +c h\left\|\delta u_{1}\right\|_{r}^{r}+\left|\left(A_{\left(0, U_{0}\right)}-A_{1}\right)\left(U_{0},\left|\delta u_{1}\right|^{r-2} \delta u_{1}\right)\right| \\
\leq & \frac{1}{r}\left\|f_{0}-A\left(0, U_{0}\right) U_{0}\right\|_{r}^{r}+\left(1-\frac{1}{r}\right)\left\|\delta u_{1}\right\|_{r}^{r} \\
& +c h\left(1+\left\|\delta u_{1}\right\|_{\mu_{3}^{\prime}(r-1)}^{r}+\left\|u_{0}\right\|_{1, r}\left\|\omega_{1}\right\|_{1,2}\left\|\omega_{1}\right\|_{s}^{(r-2) / r}\right)
\end{aligned}
$$


From this in the same way as above using the Young inequality and Lemma 2.2/b) the boundedness

$$
\left\|\omega_{1}\right\|_{2}^{2}+c h\left\|\omega_{1}\right\|_{1,2}^{2} \leq \frac{\left\|f_{0}-A(0) U_{0}\right\|_{r}^{r}+c h}{1-c h_{0}} \leq K
$$

for all $h \leq h_{0}$ follows. Therefore, (3.11) provides

$$
\left\|\omega_{i}\right\|_{2}^{2} \leq\left\|f_{0}-A(0) U_{0}\right\|_{r}^{r}+c t_{i}+c \sum_{j=1}^{i} h\left\|\omega_{j}\right\|_{2}^{2}+c \sum_{j=1}^{i-1} h\left\|\omega_{j}\right\|_{2}^{2 \beta}
$$

for $i=1, \ldots, \hat{n}$ and $h \leq h_{0}$. Hence, Lemma 2.3 applied to this nonlinear Gronwall inequality with $d_{i}=\left\|\omega_{i}\right\|_{2}^{2}$ yields (cf. also remark and corollary added to this lemma)

$$
\left\|\omega_{i}\right\|_{2}^{2}=\left\|\delta u_{i}\right\|_{r}^{r} \leq M\left(t_{i}\right)
$$

where $M\left(t_{i}\right)$ is defined as the right-hand side of (2.14). Since $\beta>1$ the bound $M(t)$ has a singularity at $t=t^{*}$, therefore assertion (3.9) follows for every fixed interval $\left[0, T^{*}\right] \subset\left[0, t^{*}\right) \cap[0, \widehat{T}]$

By the above lemma the time interval $\hat{I}$ may be reduced once more. For simplicity, however, we write $\hat{I}=[0, \widehat{T}] \cap\left[0, T^{*}\right]$ again.

Concluding this section we present two estimates (3.13) and (3.14) which are an immediate consequence of Lemma 3.3. First applying Lemma 2.2 to $\omega_{j}=\left|\delta u_{j}\right|^{(r-2) / 2} \delta u_{j}$ we get

$$
\left\|\delta u_{j}\right\|_{\nu}^{r}=\left\|\omega_{j}\right\|_{s}^{2} \leq \varepsilon\left\|\omega_{j}\right\|_{1,2}^{2}+c_{\varepsilon}\left\|\delta u_{j}\right\|_{r}^{r}
$$

and obtain from this estimate by means of (3.11), (3.12) and (3.9)

$$
\sum_{j=1}^{\grave{n}} h\left\|\delta u_{j}\right\|_{\nu}^{r} \leq c \quad \text { with } \quad \nu<\frac{r N}{N-2}
$$

Finally, in view of the a priori estimate (3.8) Lemma 3.3 yields the boundedness

$$
\left\|u_{j}\right\|_{1, r} \leq C_{3} \quad \text { for all } t_{j} \in \hat{I}
$$

of space-like derivatives. 


\section{Convergence and existence result}

In this section we deal with approximations of the solution $u$ of problem (1.1) - (1.3) defined on the cylinder $\bar{Q}_{\widehat{T}}$. Therefore we interpolate the solutions $u_{j}$ of the discretized problem (2.1) - (2.3) with respect to $t$ in the way given by (2.4) and (2.5), respectively, and obtain the piecewise linear and piecewise constant functions $\tilde{u}^{n}(x, t)$ and $\bar{u}^{n}(x, t)$, respectively. These interpolations turn out to be approximations of the weak solution $u$ of the problem (1.1) - (1.3). Moreover, using the notation

$$
\tau_{h} u(x, t)=u(x, t-h)
$$

we write

$$
\bar{f}^{n}=\hat{f}\left(\cdot, \bar{t}^{n}, \tau_{h} \bar{u}^{n}\right) \quad \text { and } \quad \bar{A}^{n}(\cdot, \cdot)=A_{\left(\bar{\imath}^{n}, \tau_{h} \bar{u}^{n}\right)}(\cdot, \cdot)
$$

with $\bar{t}^{n}=t_{j}^{n}$ if $t_{j-1}^{n}<t \leq t_{j}^{n}$. Now piecewise constant interpolation of $(2.1)$; over $\hat{I}$ yields

$$
\int_{\dot{I}}\left\langle D_{t} \bar{u}^{n}, v\right\rangle d t+\int_{\dot{I}} \bar{A}^{n}\left(\bar{u}^{n}, v\right) d t=\int_{\dot{I}}\left\langle\bar{f}^{n}, v\right\rangle d t
$$

for all $v \in L_{1}\left(\hat{I}, W_{0}^{1, r^{\prime}}(G)\right)$. The results of Section 3 may be rewritten in the following form:

$$
\begin{aligned}
& \bar{u}^{n}(\cdot, t), \tilde{u}^{n}(\cdot, t) \in \mathcal{B}_{R}\left(U_{0}\right) \\
& \left\|D_{t} \tilde{u}^{n}(\cdot, t)\right\|_{r} \leq C_{2} \text { and }\left\|\tilde{u}^{n}(\cdot, t)-\bar{u}^{n}(\cdot, t)\right\|_{r} \leq C_{2} h_{n} \\
& \int_{\bar{I}}\left\|\bar{u}^{n}(\cdot, t)-\tau_{h} \bar{u}^{n}(\cdot, t)\right\|_{\nu}^{r} d t \leq c h_{n}^{r} \\
& \left\|\bar{u}^{n}(\cdot, t)\right\|_{1, r} \leq C_{3} \text { and }\left\|\bar{u}^{n}(\cdot, t)\right\|_{1, r} \leq C_{3}
\end{aligned}
$$

for all $t \in \hat{I}$. Next we prove convergence of the Rothe approximations.

Lemma 4.1. The interpolations $\tilde{u}^{n}$ of the solutions $u_{j}$ of the discretized problem (2.1) - (2.3) converge in $C\left(\hat{I}, L_{r}(G)\right)$ to a limit function $u$ and the error estimate

$$
\left\|\tilde{u}^{n}-u\right\|_{C\left(I, L_{r}(G)\right)} \leq C_{4} h_{n}^{1 / 2}
$$

holds with some positive constant $C_{4}$.

Proof. We follow the proof of Lemma 6 in [8], therefore we only give an outline of the corresponding estimates. We want to show that $\left\{\tilde{u}^{n}\right\}$ is a Cauchy sequence in $C\left(\hat{I}, L_{r}(G)\right)$. Therefore we estimate the difference $\tilde{u}^{m, n}=\tilde{u}^{m}-\tilde{u}^{n}$. Analogously, we define $\bar{u}^{m, n}=\bar{u}^{m}-\bar{u}^{n}$ and $\bar{\omega}^{m, n}=\left|\bar{u}^{m, n}\right|^{(r-2) / 2} \bar{u}^{m, n}$.

First of all we state that

$$
\begin{aligned}
D_{t}\left\|\tilde{u}^{m, n}(\cdot, t)\right\|_{r}^{r}= & r\left\langle D_{t} \tilde{u}^{m, n},\left|\tilde{u}^{m, n}\right|^{r-2} \tilde{u}^{m, n}\right\rangle \\
\leq & r\left\langle D_{t} \tilde{u}^{m, n},\left|\tilde{u}^{m, n}\right|^{r-2} \bar{u}^{m, n}\right\rangle+c\left\|\tilde{u}^{m, n}(\cdot, t)\right\|_{r}^{r} \\
& +c\left(h_{m}+h_{n}\right)^{r}+c\left(h_{m}+h_{n}\right)^{r / 2}
\end{aligned}
$$


because of the inequality

$$
\begin{aligned}
& \left\|\left|\tilde{u}^{m, n}\right|^{r-2} \tilde{u}^{m, n}-\left|\bar{u}^{m, n} \cdot\right|^{r-2} \bar{u}^{m, n}\right\|_{r^{\prime}} \\
& \quad \leq(r-1)\left(\left\|\tilde{u}^{m, n}\right\|_{r}+\left\|\bar{u}^{m, n}\right\|_{r}\right)^{r-2}\left\|\tilde{u}^{m, n}-\bar{u}^{m, n}\right\|_{r} \\
& \quad \leq(r-1)\left(2\left\|\tilde{u}^{m, n}\right\|_{r}+C_{2}\left(h_{m}+h_{n}\right)\right)^{r-2} C_{2}\left(h_{m}+h_{n}\right),
\end{aligned}
$$

the Young inequality and the second inequality (4.3). Now we take the difference of the relations $(4.1)^{m}$ and $(4.1)^{n}$ for two different subdivisions into $m$ and $n$ subintervals, respectively, and insert the test function

$$
v(\cdot, t)= \begin{cases}\left|\bar{u}^{m, n}\right|^{r-2} \bar{u}^{m, n} & \text { if } 0 \leq t \leq t_{0} \\ 0 & \text { if } t>t_{0}\end{cases}
$$

into this difference $(4.1)^{m}-(4.1)^{n}$. The resulting equation is used to replace the first term on the right of (4.7) after an integration of (4.7) over $t \in\left[0, t_{0}\right]$. Then we obtain

$$
\begin{aligned}
\left\|\tilde{u}^{m, n}\left(\cdot, t_{0}\right)\right\|_{r}^{r} & +r \int_{0}^{t_{0}} \bar{A}^{n}\left(\bar{u}^{m, n},\left|\bar{u}^{m, n}\right|^{r-2} \bar{u}^{m, n}\right) d t \\
& \leq r \int_{0}^{t_{0}}\left(\bar{A}^{n}-\bar{A}^{m}\right)\left(\bar{u}^{m},\left|\bar{u}^{m, n}\right|^{r-2} \bar{u}^{m, n}\right) d t \\
& +r \int_{0}^{t_{0}}\left\|\bar{f}^{m}-\bar{f}^{n}\right\|_{\mu_{3}}\left\|\bar{u}^{m, n}\right\|_{\mu_{3}^{\prime}(r-1)}^{r-1} d t \\
& +c \int_{0}^{t_{0}}\left\|\tilde{u}^{m, n}\right\|_{r}^{r} d t+c\left(h_{m}+h_{n}\right)^{r / 2}
\end{aligned}
$$

In view of (2.9) and the boundedness (4.5) we have

$$
\begin{aligned}
& \left|\left(\bar{A}^{n}-\bar{A}^{m}\right)\left(\bar{u}^{m},\left|\bar{u}^{m, n}\right|^{r-2} \bar{u}^{m, n}\right)\right| \\
& \quad \leq c\left(\left(h_{m}+h_{n}\right)+\left\|\tau_{h_{n}} \bar{u}^{n}-\tau_{h_{m}} \bar{u}^{m}\right\|_{\nu}\right)\left\|\bar{u}^{m}\right\|_{1, r}\left\|\bar{\omega}^{m, n}\right\|_{1,2}\left\|\bar{\omega}^{m, n}\right\|_{s}^{(r-2) / r} \\
& \quad \leq \varepsilon\left\|\bar{\omega}^{m, n}\right\|_{1,2}^{2}+c\left(\left(h_{m}+h_{n}\right)^{r}+\left\|\tau_{h_{n}} \bar{u}^{n}-\tau_{h_{m}} \bar{u}^{m}\right\|_{\nu}^{r}+\left\|\bar{\omega}^{m, n}\right\|_{g}^{2}\right) .
\end{aligned}
$$

Now regarding the estimates (2.7), Assumption (iii), inequality (4.4) and the Young 
inequality we continue the above estimation by

$$
\begin{aligned}
\left\|\tilde{u}^{m, n}\left(\cdot, t_{0}\right)\right\|_{r}^{r}+r & k_{1} \int_{0}^{t_{0}}\left\|\bar{\omega}^{m, n}(\cdot, t)\right\|_{1,2}^{2} d t \\
\leq & \varepsilon \int_{0}^{t_{0}}\left\|\bar{\omega}^{m, n}(\cdot, t)\right\|_{1,2}^{2} d t \\
& +c \int_{0}^{t_{0}}\left(\left\|\tilde{u}^{m, n}\right\|_{r}^{r}+\left\|\bar{\omega}^{m, n}\right\|_{s}^{2}\right) d t+c\left(h_{m}+h_{n}\right)^{r / 2} .
\end{aligned}
$$

Since $s=\max \left\{\frac{2(r-1) \mu_{3}^{\prime}}{r}, \frac{2 \nu}{r}\right\}<\frac{2 N}{N-2}$ we can apply Lemma $\left.2.2 / \mathrm{b}\right)$ with $q=2$ and then (4.3). Hence, for small $\varepsilon>0$ there follows

$$
\left\|\tilde{u}^{m, n}\left(\cdot, t_{0}\right)\right\|_{r}^{r} \leq c\left(h_{m}+h_{n}\right)^{r / 2}+c \int_{0}^{t_{0}}\left\|\tilde{u}^{m, n}(\cdot, t)\right\|_{r}^{r} d t
$$

which yields by means of the usual Gronwall lemma

$$
\left\|\tilde{u}^{m}(\cdot, t)-\tilde{u}^{n}(\cdot, t)\right\|_{r}^{r} \leq c\left(h_{m}+h_{n}\right)^{r / 2} e^{c t} \quad \text { for all } t \in \hat{I}
$$

This implies that $\left\{\tilde{u}^{n}\right\}$ is a Cauchy sequence in the Banach space $C\left(\hat{I}, L_{r}(G)\right)$ which converges to $u$. Passing to the limit $m \rightarrow+\infty$ in (4.8) this yields the error estimate (4.6)

Actually, the approximations have stronger convergence than in $C\left(\hat{I}, L_{r}(G)\right)$. We may derive convergence even in Hölder spaces.

Lemma 4.2. Let $\tilde{u}^{n}$ be the interpolations introduced at the beginning of this section. Then there is an $\alpha \in \mathbb{R}$ with $0<\alpha<1-\frac{N}{r}$ such that

$$
\tilde{u}^{n} \longrightarrow u \quad \text { in } C^{\alpha}\left(\bar{Q}_{\widehat{T}}\right) \text { for } n \rightarrow+\infty
$$

Moreover, for every $\lambda \in \mathbb{R}$ with $0<\lambda<1-\frac{N}{r}$ it holds

$$
\tilde{u}^{n} \longrightarrow u \quad \text { in } C\left(\hat{I}, C^{\lambda}(\bar{G})\right) \text {. for } n \rightarrow+\infty
$$

with convergence order $O\left(h_{n}^{(1-N / r-\lambda) / 2}\right)$.

Proof. a) We start with the proof of (4.10). Therefore we apply the NirenbergGagliardo interpolation inequality

$$
\|v\|_{C^{\lambda}(\bar{G})} \leq c\|v\|_{1, r}^{\theta}\|v\|_{r}^{1-\theta} \quad \text { for } \quad v \in W_{0}^{1, r}(G) \quad\left(\lambda+\frac{N}{r} \leq \theta<1\right)
$$


to the difference $v=\tilde{u}^{m}-\tilde{u}^{n}$. Because of the boundedness (4.5) and estimate (4.8) we get

$$
\sup _{t \in \hat{I}}\left\|\tilde{u}^{m}(\cdot, t)-\tilde{u}^{n}(\cdot, t)\right\|_{C^{\lambda}(\bar{G})} \leq c\left(h_{m}+h_{n}\right)^{(1-N / r-\lambda) / 2} .
$$

This yields property (4.10). Then the sequence $\left\{\tilde{u}^{n}\right\}$ is also bounded in $C\left(\hat{I}, C^{\lambda}(\bar{G})\right)$ :

$$
\sup _{t \in \tilde{I}}\left\|\tilde{u}^{n}(\cdot, t)\right\|_{C^{\lambda}(\bar{G})} \leq c,
$$

which will be used in the next step.

b) The assertion of Lemma 3.2 implies

$$
\left\|\tilde{u}^{n}\left(\cdot, t_{j}\right)-\tilde{u}^{n}\left(\cdot, t_{k}\right)\right\|_{C(\bar{G})} \leq c_{1}\left|t_{j}-t_{k}\right|^{\lambda_{1}}
$$

if $t_{j}$ and $t_{k}$ are subdivision points. Then

$$
\left\|\tilde{u}^{n}\left(\cdot, t^{\prime}\right)-\dot{u}^{n}\left(\cdot, t^{\prime \prime}\right)\right\|_{C(\bar{G})} \leq 3^{1-\lambda_{1}} c_{1}\left|t^{\prime}-t^{\prime \prime}\right|^{\lambda_{1}}
$$

for arbitrary points $t^{\prime}, t^{\prime \prime} \in \hat{I}$ and arbitrary natural $n$. In fact, let first $t^{\prime}$ and $t^{\prime \prime}$ belong to the same subinterval $\left[t_{j-1}, t_{j}\right]$. Then

$$
\tilde{u}^{n}\left(\cdot, t^{\prime}\right)-\tilde{u}^{n}\left(\cdot, t^{\prime \prime}\right)=\left(t^{\prime \prime}-t^{\prime}\right) \frac{\tilde{u}^{n}\left(\cdot, t_{j-1}\right)-\tilde{u}^{n}\left(\cdot, t_{j}\right)}{h_{n}}
$$

hence

$$
\left\|\tilde{u}^{n}\left(\cdot, t^{\prime}\right)-\tilde{u}^{n}\left(\cdot, t^{\prime \prime}\right)\right\|_{C(\bar{G})} \leq \frac{\left|t^{\prime}-t^{\prime \prime}\right|}{h_{n}} c_{1} h_{n}^{\lambda_{1}} \leq c_{1}\left|t^{\prime \prime}-t^{\prime}\right|^{\lambda_{1}}
$$

because of $\left|t^{\prime \prime}-t^{\prime}\right| \leq h_{n}$. If now $t_{k-1}<t^{\prime} \leq t_{k} \leq t_{j-1}<t^{\prime \prime} \leq t_{j}$, then formula (4.12) follows from the triangle axiom

$$
\begin{aligned}
\| \tilde{u}^{n}\left(\cdot, t^{\prime}\right)- & \tilde{u}^{n}\left(\cdot, t^{\prime \prime}\right) \|_{C(\bar{G})} \\
\leq & \left\|\tilde{u}^{n}\left(\cdot, t^{\prime}\right)-\tilde{u}^{n}\left(\cdot, t_{k}\right)\right\|_{C(\bar{G})} \\
& +\left\|\tilde{u}^{n}\left(\cdot, t_{k}\right)-\tilde{u}^{n}\left(\cdot, t_{j-1}\right)\right\|_{C(\bar{G})}+\left\|\tilde{u}^{n}\left(\cdot, t_{j-1}\right)-\tilde{u}^{n}\left(\cdot, t^{\prime \prime}\right)\right\|_{C(\bar{G})} .
\end{aligned}
$$

Thus in view of (4.11) and (4.12) the approximations are Hölder continuous with respect to the space variable $x$ for fixed $t \in \hat{I}$, and with respect to the time variable $t$ for fixed $x \in G$, with uniformly bounded Hölder constants. Then also $\left\|\tilde{u}^{n}\right\|_{C^{a_{1}}\left(\bar{Q}_{\hat{t}}\right)} \leq c$ for all $n$ with $\alpha_{1}=\min \left\{\lambda_{1}, \lambda\right\}$. By the compact embedding $C^{\alpha_{1}}\left(\bar{Q}_{\widehat{T}}\right) \subset C^{\alpha}\left(\bar{Q}_{\widehat{T}}\right)$ for $\alpha<\alpha_{1}$ there is a subsequence $\left\{\tilde{u}^{n_{k}}\right\}$ that converges in $C^{\alpha}\left(\bar{Q}_{\widehat{T}}\right)$. Since this means in particular uniform convergence on $\bar{Q}_{\widehat{T}}$ the limit of each convergent subsequence coincides with the limit function $u$ from (4.10) and (4.6), hence the whole sequence $\left\{\tilde{u}^{n}\right\}$ converges to $u \in C^{\alpha}\left(\bar{Q}_{\widehat{T}}\right)$ 
Note that Lemma 3.2 also implies

$$
\left\|\tilde{u}^{n}(\cdot, t)-\tau_{h_{n}} \bar{u}^{n}(\cdot, t)\right\|_{C(\bar{G})} \leq\left\|u_{j}-u_{j-1}\right\|_{C(\bar{G})} \leq c h_{n}^{\lambda_{1}}
$$

hence besides

$$
\tau_{h_{n}} \bar{u}^{n} \longrightarrow u \quad \text { uniformly for all }(x, t) \in \bar{Q}_{\widehat{T}}
$$

holds, too.

The limit function $u$ of the Rothe approximations $\tilde{u}^{n}$ turns out to be a weak solution of the initial boundary value problem (1.1) - (1.3). We summarize the results in the following statement.

Theorem 4.1. Suppose Assumptions (i) - (iii). Then there is an interval $\hat{I}=[0, \widehat{T}]$ and a number $\alpha>0$ such that problem (1.1) - (1.3) has a unique weak solution $u \in$ $L_{\infty}\left(\hat{I}, W_{0}^{1, r}(G)\right) \cap C^{\alpha}\left(\bar{Q}_{\widehat{T}}\right)$, with $D_{t} u \in L_{\infty}\left(\hat{I}, L_{r}(G)\right)$ fulfilling the relation

$$
\int_{\dot{I}}\left\langle D_{t} u, v\right\rangle d t+\int_{\dot{I}} A_{(t, u)}(u, v) d t=\int_{i}\langle f, v\rangle d t
$$

for all $v \in L_{1}\left(\hat{I}, W_{0}^{1, r^{\prime}}(G)\right)$. The Rothe approximations $\tilde{u}^{n}$ and $\bar{u}^{n}$ have the convergence properties

$$
\begin{gathered}
\tilde{u}^{n} \longrightarrow u \quad \text { in } C^{\alpha}\left(\bar{Q}_{\widehat{T}}\right) \cap C\left(\hat{I}, C^{\lambda}(\bar{G})\right) \\
\bar{u}^{n} \longrightarrow u \quad \text { in } L_{\infty}\left(\hat{I}, C^{\lambda}(\bar{G})\right) \quad\left(\lambda<1-\frac{N}{r}\right) \\
\tilde{u}^{n}, \bar{u}^{n} \longrightarrow u \quad \text { in } L_{\infty}\left(\hat{I}, W_{0}^{1, p}(G)\right) \quad(p<r) \\
\tilde{u}^{n}, \bar{u}^{n} \neq u \quad \text { in } L_{\infty}\left(\hat{I}, W_{0}^{1, r}(G)\right) \\
D_{t} \tilde{u}^{n} \neq D_{\imath} u \quad \text { in } L_{\infty}\left(\hat{I}, L_{r}(G)\right)
\end{gathered}
$$

as $n$ tends to infinity.

Proof. a) We start with the proof of the convergence properties. Formula (4.15) is the assertion of Lemma 4.2. Because of (4.3) and (4.6), for the approximations $\bar{u}^{n}$ being non-continuous and piecewise constant with respect to $t$, an estimate as (4.6),

$$
\sup _{t \in \dot{I}}\left\|\bar{u}^{n}(\cdot, t)-u(\cdot, t)\right\|_{r} \leq c h_{n}^{1 / 2}
$$

holds. By the same computations as in the proof of (4.10) that yields (4.16).

In order to prove (4.17) we take the difference of the relations $(4.1)^{m}-(4.1)^{n}$ (without integration over $t$ ) applied to the test function $v=\bar{u}^{m}-\bar{u}^{n}$, which gives

$$
\begin{aligned}
& \left\langle D_{\imath}\left(\tilde{u}^{m}-\tilde{u}^{n}\right), \bar{u}^{m}-\bar{u}^{n}\right\rangle+\bar{A}^{m}\left(\bar{u}^{m}-\bar{u}^{n}, \bar{u}^{m}-\bar{u}^{n}\right) \\
& =\left\langle\bar{f}^{m}-\bar{f}^{n}, \bar{u}^{m}-\bar{u}^{n}\right\rangle+\left(\bar{A}^{n}-\bar{A}^{m}\right)\left(\bar{u}^{n}, \bar{u}^{m}-\bar{u}^{n}\right),
\end{aligned}
$$


and estimate it applying (2.7) and (2.9) with $p=2$ and then (4.3), (3.7) and (4.5) as well as the Young inequality. This leads to

$$
\begin{aligned}
k_{1} \| \bar{u}^{m}- & \bar{u}^{n} \|_{1,2}^{2} \\
\leq & k_{2}\left\|\bar{u}^{m}-\bar{u}^{n}\right\|_{2}^{2} \\
& +\left(\left\|D_{t} \tilde{u}^{m}\right\|_{r}+\left\|D_{t} \tilde{u}^{n}\right\|_{r}+\left\|\bar{f}^{m}\right\|_{r}+\left\|\bar{f}^{n}\right\|_{r}\right)\left\|\bar{u}^{m}-\bar{u}^{n}\right\|_{r^{\prime}} \\
& +c\left(\left(h_{m}+h_{n}\right)+\left\|\tau_{h_{m}} \bar{u}^{m}-\tau_{h_{n}} \bar{u}^{n}\right\|_{\nu}\right)\left\|\bar{u}^{n}\right\|_{1, r}\left\|\bar{u}^{m}-\bar{u}^{n}\right\|_{1,2} \\
\leq & \varepsilon\left\|\bar{u}^{m}-\bar{u}^{n}\right\|_{1,2}^{2} \\
& +c\left(\left\|\bar{u}^{m}-\bar{u}^{n}\right\|_{C(\bar{G})}+\left(h_{m}+h_{n}\right)^{2}+\left\|\tau_{h_{m}} \bar{u}^{m}-\tau_{h_{n}} \bar{u}^{n}\right\|_{C(\bar{G})}^{2}\right)
\end{aligned}
$$

for all $t \in \hat{I}$. Then the uniform convergences (4.16) and (4.13) yield

$$
\sup _{t \in \tilde{I}}\left\|\bar{u}^{m}(\cdot, t)-\bar{u}^{n}(\cdot, t)\right\|_{1,2} \longrightarrow 0 \quad \text { as } m, n \rightarrow+\infty
$$

Assertion (4.17) for the sequence $\left\{\bar{u}^{n}\right\}$ then follows from the interpolation inequality

$$
\|v\|_{p} \leq c\|v\|_{r}^{\theta}\|v\|_{2}^{1-\theta} \quad\left(2 \leq p<r, \frac{1}{p}=\frac{\theta}{r}+\frac{1-\theta}{2}\right)
$$

between Lebesgue spaces applied to $v=\nabla\left(\bar{u}^{m}-\bar{u}^{n}\right)$ using the boundedness (4.5). In order to prove (4.17) for the sequence $\left\{\tilde{u}^{n}\right\}$ piecewise linear interpolation of the relations (2.1); instead of $(4.1)^{n}$ has to be used to obtain its convergence in $L_{\infty}\left(\hat{I}, W_{0}^{1,2}(G)\right)$. Then interpolation as above yields the assertion. We omit detailed calculations.

Property (4.18) follows from (4.5) because of the weak* compactness of bounded sequences in $L_{\infty}(\hat{I}, X)$. This yields weak* convergence for a subsequence, however since the limit must be the same function $u$ for every weak* convergent subsequence the whole sequence converges. By the same argument, the first estimate in (4.3) implies the convergence (4.19).

b) A limit process $n \rightarrow+\infty$ in relation $(4.1)^{n}$ by means of the convergence properties (4.19), (4.18) and (4.13) and the Lipschitz conditions in Assumptions (ii) and (iii) immediately yields relation (4.14), i.e. the limit function $u$ is a weak solution of the differential equation (1.1). The solution fulfils the boundary condition (1.2) since it belongs to $L_{\infty}\left(\hat{I}, W_{0}^{1, r}(G)\right)$, and it fulfils the initial condition (1.3) due to the construction of the approximations $\tilde{u}^{n}$ and their uniform convergence.

Uniqueness is proved in a similar way as convergence in Lemma 4.1. Let $u^{*}$ and $u^{* *}$ be two weak solutions of $(1.1)-(1.3)$, take the difference of the corresponding two relations $(4.14)$ and insert the test function

$$
v(\cdot, t)= \begin{cases}\left|u^{*}(\cdot, t)-u^{* *}(\cdot, t)\right|^{r-2}\left(u^{*}(\cdot, t)-u^{* *}(\cdot, t)\right) & \text { if } 0 \leq t \leq t_{0} \\ 0 & \text { otherwise }\end{cases}
$$


into the resulting relation. Using the abbreviation $u=u^{*}-u^{* *}$ we obtain

$$
\begin{aligned}
\int_{0}^{t_{0}}\left\langle u,|u|^{r-2} u\right\rangle d t & +\int_{0}^{t_{0}} A_{\left(t, u^{*}\right)}\left(u,|u|^{r-2} u\right) d t \\
& =\int_{0}^{t_{0}}\left\langle f\left(\cdot, t, u^{*}\right)-f\left(\cdot, t, u^{* *}\right),|u|^{r-2} u\right\rangle d t \\
& +\int_{0}^{t_{0}}\left(A_{\left(t, u^{* *}\right)}-A_{\left(t, u^{*}\right)}\right)\left(u^{* *},|u|^{r-2} u\right) d t
\end{aligned}
$$

We denote $w=|u|^{(r-2) / 2} u$ and estimate the above equation with the aid of (2.7) and (2.9), and with Assumptions (ii) and (iii). This leads to

$$
\begin{aligned}
\left\|u\left(\cdot, t_{0}\right)\right\|_{r}^{r} & +k_{1} r \int_{0}^{t_{0}}\|w\|_{1,2}^{2} d t \\
\leq & k_{2} r \int_{0}^{t_{0}}\|u\|_{r}^{r} d t+l_{3} r \int_{0}^{t_{0}}\|u\|_{\nu}\|u\|_{r}^{r-1} d t \\
& +c \int_{0}^{t_{0}}\|u\|_{\nu}\left\|u^{* *}\right\|_{1, r}\|w\|_{1,2}\|w\|_{s}^{(r-2) / r} d t \\
\leq & \varepsilon \int_{0}^{t_{0}}\|w\|_{1,2}^{2} d t+c \int_{0}^{t_{0}}\left(\|u\|_{r}^{r}+\|w\|_{2 \nu / r}^{2}+\|w\|_{s}^{2}\right) d t \\
\leq & 2 \varepsilon \int_{0}^{t_{0}}\|w\|_{1,2}^{2} d t+c_{\varepsilon} \int_{0}^{t_{0}}\|u(\cdot, t)\|_{r}^{r} d t .
\end{aligned}
$$

In this estimation Lemma 2.2 with $\alpha=1$ and $q=2$ was used. For small $\varepsilon>0$ the Gronwall lemma yields $\|u(\cdot, t)\|_{r}=0$ for all $t \in \hat{I}$, which means $u^{*}=u^{* *}$, i.e. uniqueness of the solution of our problem in the sense of (4.14)

\section{References}

[1] Alikakos, N. D.: $L_{p}$-bounds of solutions of reaction-diffusion equations. Comm. Part. Diff. Equ. 4 (1979), $827-868$.

[2] Filo, J. and J. Kaciur: Local existence of general nonlinear parabolic systems. Nonlin. Anal.: Theory Methods Appl. 24 (1995), 1597 - 1618.

[3] Kačur, J.: Method of Rothe in Evolution Equations (Teubner-Texte zur Mathematik: Vol. 80). Leipzig: B.G. Teubner Verlagsges. 1985. 
[4] Kačur, J.: On a solution of degenerate elliptic-parabolic systems in Orlicz-Sobolev spaces. Parts I and II. Math. Z. 203 (1990), 153 - 171 and $569-579$.

[5] Kačur, J. and S. Luckhaus: Approximation of degenerate parabolic systems by nondegenerate elliptic and parabolic systems. Preprint. Bratislava: Comenius University, Faculty of Mathematics and Physics, Preprint No. M2-91 (1991), 1 - 33.

[6] Ladyżenskaja, O. A., Solonnikov, V. A. and N. N. Ural'ceva: Linear and Quasilinear Equations of Parabolic Type. Moscow: Nauka 1967.

[7] Moser, J.: A new proof of De Giorgi's theorem concerning the regularity problem for elliptic differential equations. Comm. Pure Appl. Math. 13 (1960), 457 - 468.

[8] Pluschke, V.: Local solution of parabolic equations with strongly increasing nonlinearity by the Rothe method. Czech. Math. J. 38 (1988), $642-654$.

[9] Pluschke, V.: L Lo-estimates and uniform convergence of Rothe's method for quasilinear parabolic differential equations. In: Direct and Inverse Boundary Value Problems (eds.: R. Kleinman et al). Frankfurt (M.): Peter Lang Verlag 1991, pp. 187 - 199.

[10] Pluschke, V.: Rothe's method for semilinear parabolic problems with degeneration. Math. Nachr. 156 (1992), 283 - 295.

[11] Simader, C. G.: On Dirichlet's Boundary Value Problem. Lect. Notes Math. 268 (1972), $1-238$.

[12] Willett, D. and J. Wong: On the discrete analogues of some generalizations of Gronwall's inequality. Monatsh. Math. 69 (1965), $362-367$.

Received 29.06.1995; in revised form 21.02.1996 